doi:10.1515/jrp-2015-0001

\title{
Shapiro steps in the commensurate structures with integer value of the winding number
}

\author{
Petar Mali ${ }^{1}$ and Jasmina Tekić ${ }^{2}$ \\ ${ }^{1}$ Department of Physics, Faculty of Science, University of Novi Sad, Trg Dositeja \\ Obradovića 4, 21000 Novi Sad, Serbia \\ 2 "Vinča" Institute of Nuclear Sciences, Laboratory for Theoretical and Condensed \\ Matter Physics 020, University of Belgrade, PO Box 522, 11001 Belgrade, Serbia
}

Received: September 20, 2014

\begin{abstract}
Dynamical mode locking phenomena and the appearance of Shapiro steps are studied in commensurate structures with integer values of winding number in the dc- and ac-driven overdamped Frenkel-Kontorova model. While in the standard case with sinusoidal substrate potential, the system reduces to the single particles model in which only harmonic steps exist and analytical form for the step size can be revealed, in the case of deformable potential, the presence of many degrees of freedom strongly influences the Shapiro steps. Whole series of subharmonic steps appear, and the two types of response functions, the one for the commensurate structures with odd and the one for the commensurate structures with even winding number have been observed.
\end{abstract}

Key words: Frenkel - Kontorova model, Shapiro steps, asymmetric deformable potential.

\section{Introduction}

Dynamical mode-locking and the Shapiro steps have been the subject of extensive theoretical and experimental studies in numerous physical systems such as charge-density or spin-density wave conductors $[1,2,3,4,5]$, vortex lattices $[6,7]$, Josephson-junction arrays biased by external currents $[8,9,10]$ and in recent years even superconducting nanowires $[11,12]$. Due to the great complexity of all these systems, the attention has always been focused on the simple many-body models among which the Frenkel-Kontorova (FK) model $[13,14]$ is one of the simplest but still complex enough to capture the essence of many physical and biological phenomena. Numerous results as well as still existing controversies about the existence and behavior of Shapiro steps in these highly dissipative systems particularly stimulate the studies of dissipative (overdamped) dynamics of the FK model.

The one-dimensional standard FK model represents a chain of harmonically interacting particles subjected to the sinusoidal substrate potential $[13,14]$. It can describe different 
commensurate or incommensurate structures that show very rich dynamical behavior when they are subjected to an external driver. In the presence of an external dc+ac driving force, the dynamics is characterized by the appearance of the staircase macroscopic response or the Shapiro steps in the response function $\bar{v}(\bar{F})$ of the system $[15,16,17]$. These steps appear due to the dynamical mode-locking of the internal frequency that comes from the motion of particles over the periodic substrate potential with the frequency of an external ac force. The steps are called harmonic if the locking appears for integer values of the frequency, while for the locking at noninteger rational values they are called subharmonic.

The origin of subharmonic steps has been a matter of many debates [18]. In Ref. $[20,21]$ it was proved that subharmonic steps do not exist for integer values of the winding number. For rational noninteger values of the winding number, numerical evidence of existence of subharmonic steps in the standard FK model has been found $[19,16]$ but their size is too small which makes the analysis of their properties very difficult. However, in the physical situations, such as charge-density waves, Josephson junctions, or crystals with dislocations, the application of the standard FK model could be very restricted, and it is hard to believe that real physical systems could be "exactly" described by standard models or by employing perturbation methods. Introducing a new family of nonlinear periodic deformable potentials and choosing the adequate parameters, Remoissenet and Peyrard [22] obtained a rich variety of deformable potentials in a controlled manner. These potentials allow the modeling of many specific physical situations without employing perturbation methods. They have shown that the shape of the substrate potential was of great importance for the modeling of discrete systems [22].

In this paper, we will examine the dynamical mode-locking phenomena in the commensurate structures with integer value of the winding number. We will consider two particular cases: the standard FK model with sinusoidal potential and the FK model with deformable potential. The appearance of Shapiro steps is analyzed in detail.

The paper is organized as follows. The model is introduced in Sec. 2. The results are presented and analyzed in Sec. 3, where the standard case is discussed in Sec. 3.1, and the case with deformable potential in Sec. 3.2. Finally, Sec. 4 concludes the paper.

\section{Model}

We consider the dissipative (overdamped) dynamics of a series of coupled harmonic oscillators $u_{l}$ subjected to a sinusoidal substrate (pinning) potential as follows:

$$
V(u)=\frac{K}{(2 \pi)^{2}}[1-\cos (2 \pi u)],
$$

where $K$ is the pinning strength. The system is driven by dc and ac forces:

$$
F(t)=\bar{F}+F_{a c} \cos \left(2 \pi \nu_{0} t\right) .
$$

where $F_{a c}$ and $\nu_{0}$ represent the amplitude and frequency of the ac force respectively. The equations of motions are

$$
\dot{u}_{l}=u_{l+1}+u_{l-1}-2 u_{l}-V^{\prime}\left(u_{l}\right)+F(t),
$$

where $l=-\frac{N}{2}, \ldots, \frac{N}{2}$. 
In order to generalize the model and to consider the more realistic situation, in Eq. 3, we will replace the sinusoidal potential $V\left(u_{l}\right)$ given by Eq. 1 with the one from the family of the parametrized deformable periodic potentials, the asymmetric deformable potential (ASDP) [22]:

$$
V(u)=\frac{K}{(2 \pi)^{2}} \frac{\left(1-r^{2}\right)^{2}[1-\cos (2 \pi u)]}{\left[1+r^{2}+2 r \cos (\pi u)\right]^{2}},
$$

where $r$ is the shape parameter $(-1<r<1)$. In Fig. 1, the ASDP is presented for different value of $r$.
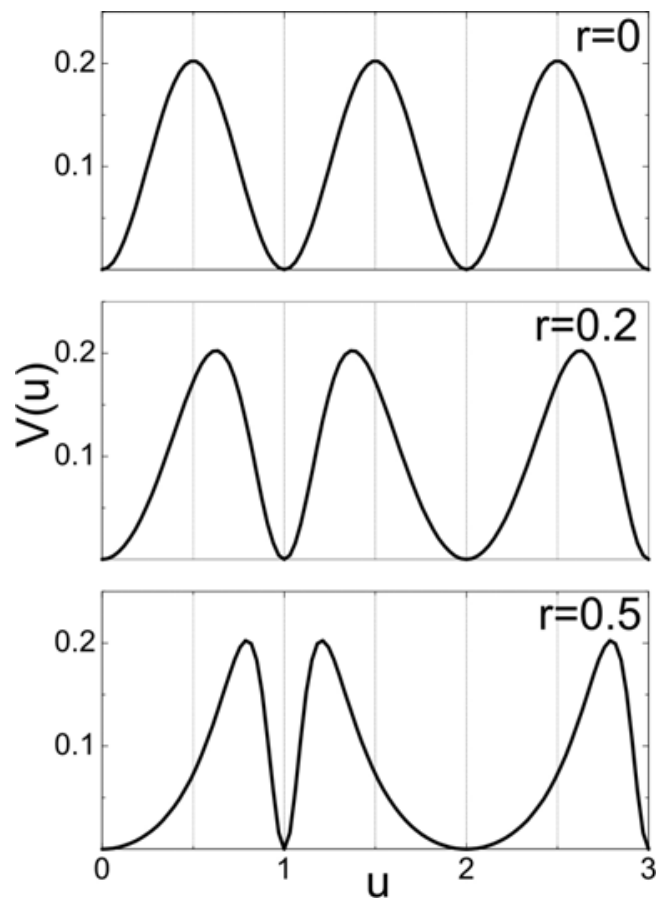

Figure 1. The substrate potential for $K=4$ and different values of the shape parameter $r$.

This potential refers to the same physical systems as the overdamped FK model [22]. By an appropriate choice of the shape parameter, it can be tuned in a controlled manner from simply periodic and symmetric sinusoidal (standard) potential for $r=0$ to an asymmetric periodic one for $0<|r|<1$ with a constant barrier height and two inequivalent successive wells with a flat and sharp bottom, respectively. The position $u_{b}$ of the potential barrier is determined by the relation $\cos \left(\pi u_{b}\right)=2 r /\left(1+r^{2}\right)$. More precisely, here the asymmetry of the substrate potential means that the pining in the two successive potential minima is different. The model has two energetically equivalent ground states, but these two states are not physically equivalent, in particular, they do not have the same dynamical properties [22].

When the system is driven by a homogenous periodic force, the competition between the two frequency scales (the frequency $\nu_{0}$ of the external periodic force and the characteristic frequency of the motion over the periodic substrate potential driven by the average force $\bar{F}$ ) can result in the appearance of the synchronization phenomena (resonance). The ac force induces an additional polarization energy into the system that is different from 
zero (less than zero) only when the velocity reaches the resonant values [15]:

$$
\bar{v}=\frac{i \omega+j}{m} \nu_{0},
$$

where $i, j$ and $m$ are integers ( $m=1$ for harmonic, and $m>1$ for subharmonic steps). In the same time, the average pinning force will also be different from zero, and the system will get locked since the average pinning energy of the locked state (on the step) is lower than of the unlocked state. As $\bar{F}$ increases, the particles will stay locked until the pinning force can cancel the increase of $\bar{F}$.

Equation (3) has been integrated using the fourth order Runge-Kutta method with the periodic boundary conditions for the commensurate structure with the integer value of average interparticle distance (winding number) $\omega=\left\langle\left(u_{l+1}-u_{l}\right)\right\rangle$ ( $\omega$ is rational for the commensurate and irrational for the incommensurate structures). In general, for commensurate structures, $\omega$ is presented by the ratio $\frac{l}{q}$ which means that there are $q$ particles per $l$ potential minima. The time step used in the simulations was $0.02 \tau$, and a time interval of $100 \tau$ was used as a relaxation time to allow the system to reach the steady state. The force was varied with the step of $10^{-4}$. The response function $\bar{v}(\bar{F})$, the Shapiro steps in particular, are analyzed in the standard and the FK model with a deformable potential.

\section{Results}

Though the standard FK model represents the system with many degrees of freedom, in the case of commensurate structures with integer values of winding number it is reduced to a single particle or single coordinate model. We will first examine the appearance of Shapiro steps in the single particle case (standard FK model), and then, extend our analysis to the model with deformable potential in which case the system possesses many degrees of freedom and can not be reduced to the single particle model.

\subsection{The Shapiro steps in a single particle system}

We consider the standard FK model with the commensurate structures with integer value of the winding number. The winding number defines the number of particles per potential well, and the commensurate structure $\omega=1$ represents the simplest case in which there is exactly one particle per one well. The case $\omega=2,3, \ldots n$ means that there is one particle in every second, third or n-th well, respectively. In Fig. 2, the response functions $\bar{v}(\bar{F})$ for the four different commensurate structures $\omega=1,2,3$ and 4 is presented.

As can be seen, regardless of the value of $\omega$, for all commensurate structures we have the same response function. This results is the consequence of the fact that in the standard FK model, all cases are reduced to the single particles case, and therefore, the results must be the same.

In the examination of the ac-driven systems, the main interest is always focused on the existence and robustness (structural stability) of the resonant solutions against the changing of the system parameters. The influence of the amplitude of the ac force on the Shapiro steps was for years the subject of experimental and theoretical studies in various systems such as charge density wave conductors $[2,3,4]$ or systems of Josephson junction arrays $[9,23,24,25,26]$. It is well known that in the standard FK model, the width of the Shapiro steps and the critical depinning force exhibits Bessel like oscillations with 
the amplitude of the ac force where maxima of one curve corresponds to the minima of another $[2,3,4]$.

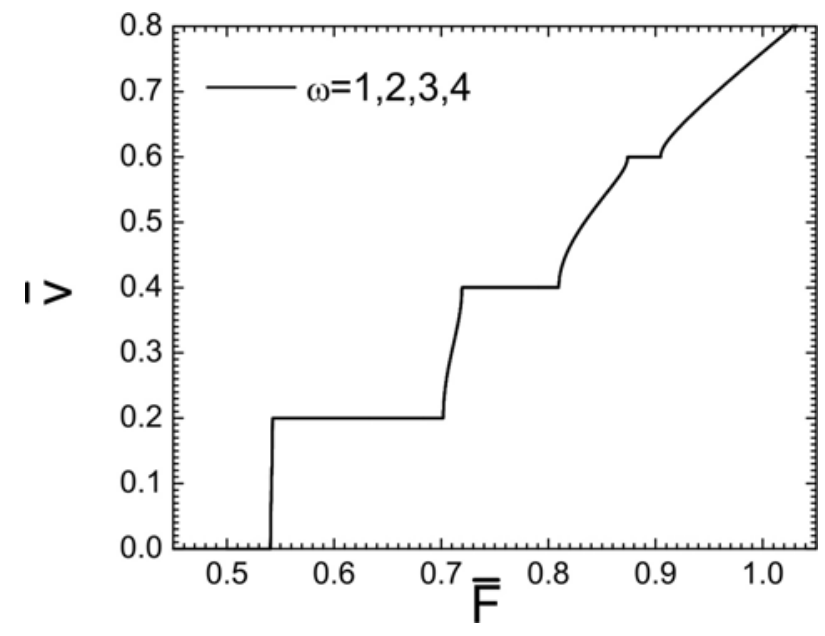

Figure 2. Average velocity as a function of the average driving force for $K=4, F_{a c}=0.2$, $\nu_{0}=0.2$, and $\omega=1,2,3$ and 4.

In Fig. 3, the width $\Delta F$ of the first harmonic step and the critical depinning force $F_{c}$ as a function of the ac amplitude $F_{a c}$ are presented.

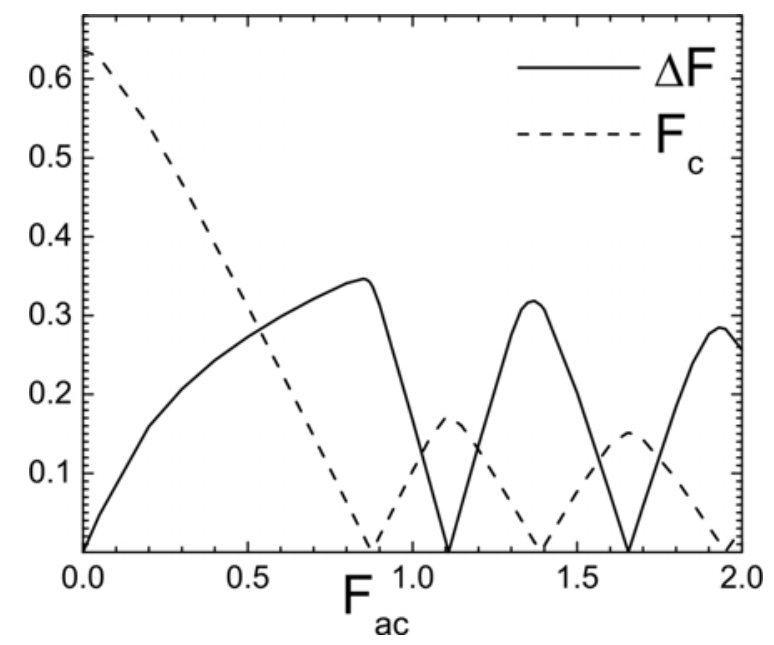

Figure 3. The width of the first harmonic step $\bar{v}=\omega \nu_{0}$ as a function of the ac amplitude for $K=4, \nu_{0}=0.2$, and $\omega=1,2,3, \ldots$

The size of a step exhibits Bessel like oscillations with the ac amplitude due to the back and forward displacement of particles induced by the ac force, where the ac amplitude determines how much this motion is retarded [2, 4, 27]. For the values of the ac amplitude that correspond to the first maximum, the particles will spend most of the time pinned, and then hop to the next well, while for the values at the second maximum, the particles will jump one site back and two forward. As the ac amplitude increases, the particles will hop between the wells that are more and more distant while staying less and less time pinned, and consequently, the step width will decrease. 
Since the FK model with integer value of $\omega$ reduces to the single particles model, Bessel like oscillations of the step size can be revealed analytically as well. In case of integer $\omega$, equation Eq. 3 becomes:

$$
\dot{u}=-V^{\prime}(u)+F(t)=-V^{\prime}(u)+\bar{F}+F_{a c} \cos \left(2 \pi \nu_{0} t\right) .
$$

Although $V^{\prime}(u)$ changes from point to point in space, the term $-V^{\prime}(u)+\bar{F}$ is constant for each period of the potential $a_{S}$ (the equal time the particle need to get from point 1 to point 2, as to get from point 2 to point 3, see Fig. 4), and it corresponds to a drift velocity $v_{d}$. It holds when $\bar{F}$ is greater then $\max \left[V^{\prime}(u)\right]$.

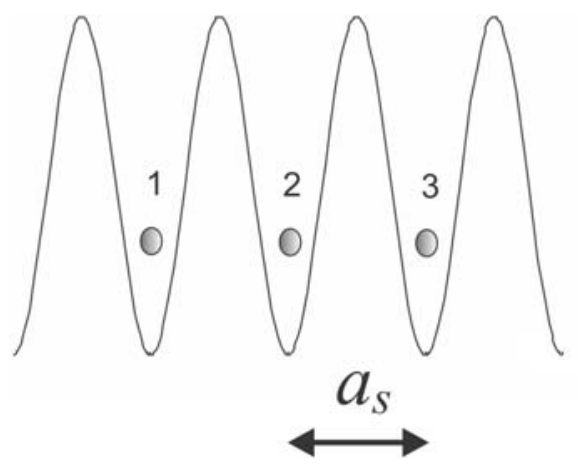

Figure 4. Position of the particles in the commensurate structure $\omega=1$.

Using the drift velocity, equation (6) can be written in the form:

$$
\dot{u}=-v_{d}+F_{a c} \cos \left(2 \pi \nu_{0} t\right) .
$$

which leads to

$$
u(t)=-v_{d} t+\frac{F_{a c}}{2 \pi \nu_{0}} \sin \left(2 \pi \nu_{0} t\right)+u_{0},
$$

where $u_{0}$ is the integration constant. In the case when the substrate potential is a periodic and an even function, it can be written as:

$$
V(u)=\frac{a_{0}}{2}+\sum_{q=1}^{\infty} a_{q} \cos (q \alpha u) .
$$

where $\alpha=1$ or 2 for pinning periodicities of $2 \pi$ or $\pi$, respectively. Substituting (8) in (9), and using the identity

$$
\exp \left[-i \frac{F_{a c}}{2 \pi \nu_{0}} \sin \left(2 \pi \nu_{0} t\right)\right]=\sum_{p=-\infty}^{\infty} J_{p}\left(\frac{F_{a c}}{2 \pi \nu_{0}}\right) \exp \left(-i p 2 \pi \nu_{0} t\right)
$$

yields

$$
V(u)=\frac{a_{0}}{2}+\sum_{q=1}^{\infty} \sum_{p=-\infty}^{\infty} a_{q} J_{p}\left(\frac{q \alpha F_{a c}}{2 \pi \nu_{0}}\right) \cos \left[\left(2 \pi p \nu_{0}-q \alpha v_{d}\right) t+q \alpha u_{0}\right],
$$

where $a_{q}$ is the $q$ th Fourier component, and $J_{p}$ is the $p$ th order Bessel function. We will assume that mode locking occurs when the time average pinning energy in the locked state is lower than in the unlocked state. From equation (11) the time-average pinning energy 
is $\langle V(u)\rangle=\frac{a_{0}}{2}$, unless in case of resonance $2 \pi p \nu_{0}=q \alpha v_{d}$ when there is an additional polarization energy:

$$
\delta\left\langle V\left(u_{0}\right)\right\rangle=\sum_{n} a_{q} J_{p}\left(\frac{F_{a c}}{2 \pi \nu_{0}}\right) \cos \left(q \alpha u_{0}\right),
$$

where the sum is over all $p$ and $q$ such that $\frac{p}{q}=\frac{\alpha v_{d}}{2 \pi \nu_{0}}$. Similarly, the time averaged pinning force $\delta\left\langle V^{\prime}\left(u_{0}\right)\right\rangle$ will be different from zero only when $2 \pi p \nu_{0}=q \alpha v_{d}$ in which case

$$
\delta\left\langle V^{\prime}\left(u_{0}\right)\right\rangle=\sum_{n} q \alpha a_{q} J_{p}\left(\frac{F_{a c}}{2 \pi \nu_{0}}\right) \cos \left(q \alpha u_{0}\right) .
$$

Through variation of $u_{0}$ over the range for which the polarization energy is less than zero, the pinning force adjusts to cancel the increase of velocity from the dc force, so that the average velocity of particles remains constant. This region of force for which the velocity remains constant determines the size of the Shapiro steps. Therefore, the width of the steps as a function of the ac force amplitude will also be a Bessel like function. Since $\delta\left\langle V^{\prime}\left(u_{0}\right)\right\rangle$ is an odd function of $u_{0}$ and its extremal values appear for $u_{0}=u_{m}$, the step width is proportional to $\delta\left\langle V^{\prime}\left(u_{m}\right)\right\rangle$. Note that if $V(u)=\frac{K}{(2 \pi)^{2}}[1-\cos (2 \pi u)], \delta\langle V(u)\rangle \neq 0$ only for $q=1$ and subharmonic steps are not predicted. Subharmonic steps with $q>1$ arise from the harmonics $(q>1)$ in the Fourier expansion.

\subsection{The Shapiro steps in a system with deformable potential}

When the substrate potential gets deformed, commensurate structures with integer values of the winding number can not be reduced any more into a single particle or single coordinate systems. The position of the particles in ASDP for different commensurate structures is given in Fig. 5

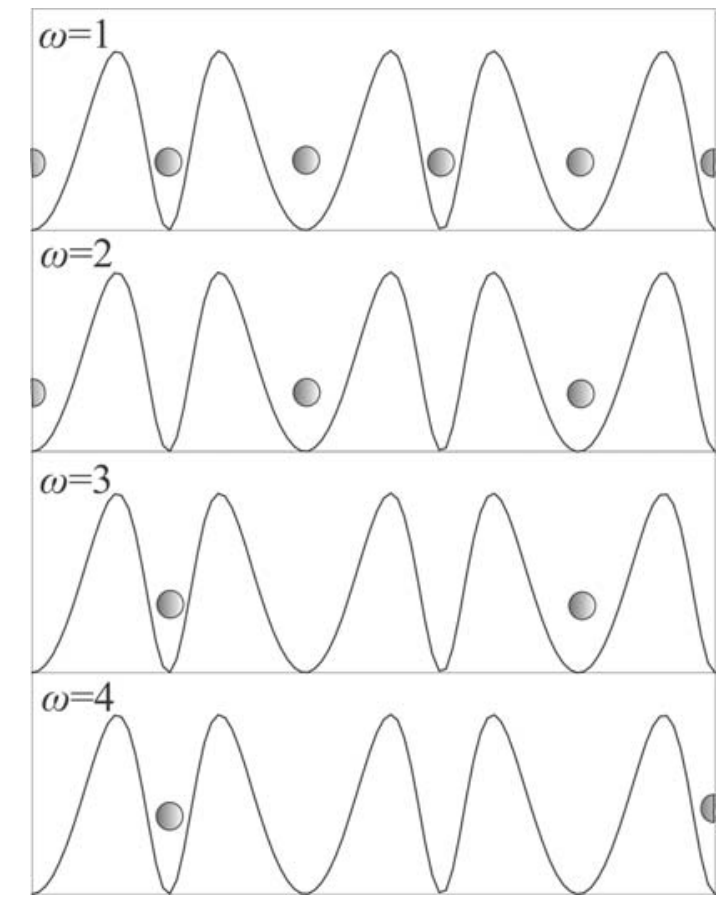

Figure 5. Position of the particles in ASDP for $\omega=1,2,3$, and 4 . 
Contrary to the standard case, the deformation of the potential will cause the appearance of subharmonic steps on the regular plot of the response function. In Fig. 6, the response functions $\bar{v}(\bar{F})$ for different commensurate structures are presented.
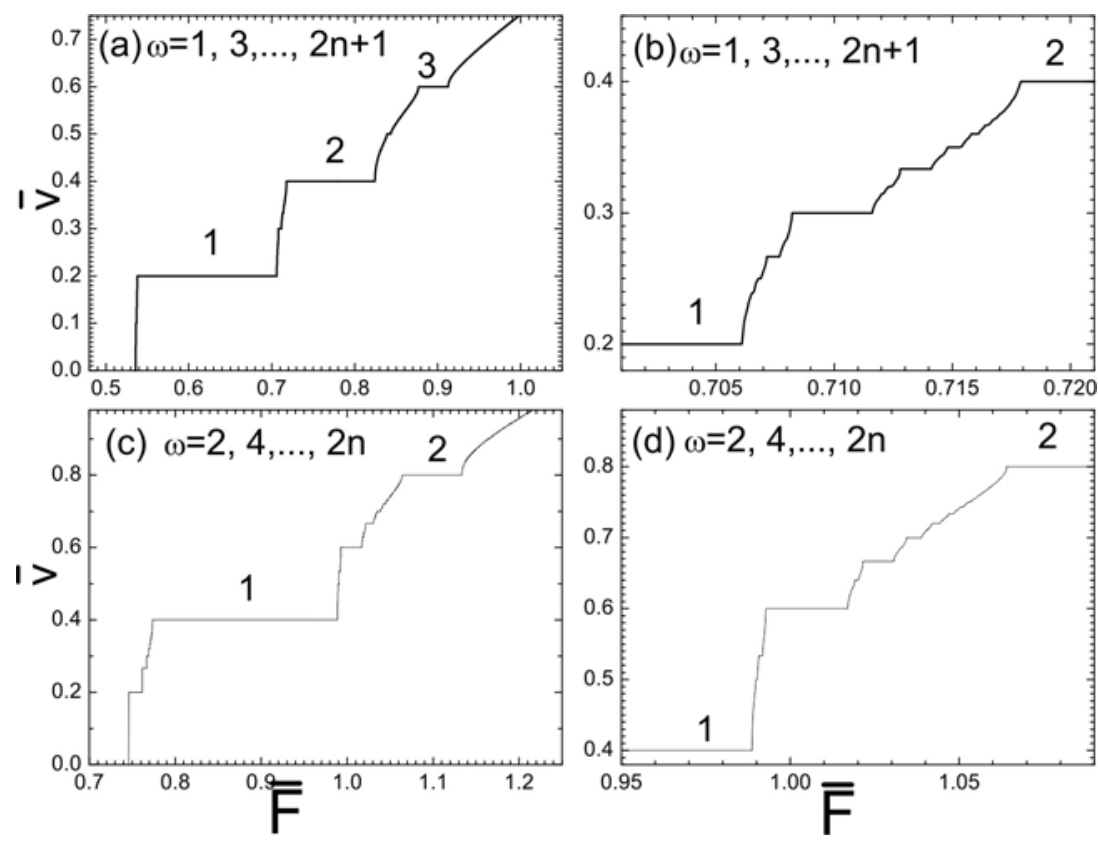

Figure 6. The average velocity as a function of the average driving force for $K=4, F_{a c}=0.2$, $\nu_{0}=0.2$, and $\omega=1,3, \ldots, 2 n+1$ in (a) and (b), and $\omega=2,4, \ldots, 2 n$ in (c) and (d). The figures in (b) and (d) correspond to the enlarged curves between the first and the second step in (a) and (c) respectively.

As can be seen in Fig. 6 (a) and (c), particularly on the enlarged curves between the first and second step in (b) and (d), whole series of subharmonic steps appear. Working on different commensurate structures, we observed that all results can be reduced into two types:

- the response function for commensurate structures with odd values of the winding number $\omega=1,3, \ldots, 2 n+1$ in Fig. 6 (a) and (b),

- the response function for commensurate structures with even values of the winding number $\omega=2,4, \ldots, 2 n$ in Fig. 6 (c) and (d).

The reason why these two types of behavior appear can be understood if we look at Fig. 5.

In THE case when $\omega$ is an even number, all particles will always be in the same well. They are either in the sharp one or in the wide one but in any case, they are equivalent. However, if the number of particles per potential is odd, while particle $i$ is in the sharp minimum, the next one $i+1$ will be in the wide one followed by the particle $i+2$ in the sharp well. Since the particles in different minima have different dynamical properties, consequently the response functions for odd or even values of $\omega$ will be different.

In Fig. 6, we can also see that this has a strong influence on the size of the Shapiro steps and the critical depinning force. Detailed study on how the size of different steps and their mutual correspondence change with the deformation will be published elsewhere. 


\section{Conclusion}

In this paper we have presented a study of the dynamical mode locking phenomena in the commensurate structures with integer value of the winding number of an overdamped dc+ac driven FK model. The presented results had shown that in the standard FK model, the system is reduced to the single particle model and consequently, the same response function has been observed for all commensurate structures. In the single particle case, only harmonic steps appear, and the analytical form for the steps size can be obtained. However, contrary to the standard case, when the potential gets deformed, the whole series of subharmonic steps appear. Since particles in different potential wells have different dynamical properties, commensurate structures with odd and even winding numbers will have different behavior and different response functions.

The presented results could be important for the studies of all real systems closely related to the dissipative dynamics of the FK model $[1,15,16,17]$, such as studies of the charge- or spin-density waves systems $[2,3,4]$ and the systems of Josephson-junction arrays $[8,9,23,24,25,26]$. In spite of numerous experimental and theoretical results in these systems, there is still no satisfactory description of the mechanism behind the interference phenomena, particularly the subharmonic mode locking. The origin of the subharmonic Shapiro steps is still a matter of debates. We hope these results could contribute to the understanding of interference phenomena in these complex physical systems, and bring a new insight into the theory of the Shapiro steps.

\section{Acknowledgments}

This work was supported by the Serbian Ministry of Education and Science under Contracts No. III-45010 and No. OI-171009.

\section{References}

[1] G. Grüner, Rev. Mod. Phys. 60, 1129 (1988).

[2] R. E. Thorne, J. S. Hubacek, W. G. Lyons, J. W. Lyding, and J. R. Tucker, Phys. Rev. B 37, 10055 (1988).

[3] R. E. Thorne, W. G. Lyons, J. W. Lyding, J. R. Tucker, and J. Bardeen, Phys. Rev. B 35, 6348 (1987).

[4] R. E. Thorne, W. G. Lyons, J. W. Lyding, J. R. Tucker, and J. Bardeen, Phys. Rev. B 35, 6360 (1987).

[5] G. Kriza et al., Phys. Rev. Lett. 66, 1922 (1991).

[6] N. Kokubo, R. Besseling, V. M. Vinokur, and P. H. Kes, Phys. Rev. Lett. 88, 247004 (2002).

[7] A. B. Kolton, D. Domínguez, and N. Grønbech-Jensen, Phys. Rev. Lett. 86, 4112 (2001). 
[8] S. P. Benz, M. S. Rzchowski, M. Tinkham, and C. J. Lobb, Phys. Rev. Lett. 64, 693 (1990).

[9] H. Sellier, C. Baraduc, F. Lefloch, and R. Calemczuk, Phys. Rev. Lett. 92, 257005 (2004).

[10] J. U. Free et al., Phys. Rev. B 41, 7267 (1990).

[11] R. C. Dinsmore III, M. H. Bae, and A. Bezryadin Appl. Phys. Lett. 93, 192505 (2008).

[12] M. H. Bae, R. C. Dinsmore III, T. Aref, M. Brenner, and A. Bezryadin Nano Lett. 9, 1889 (2009).

[13] O. Braun and Yu. S. Kivshar, The Frenkel-Kontorova Model (Springer, Berlin, 2003).

[14] O. Braun and Yu. S. Kivshar, Phys. Rep. 306, 1 (1998).

[15] L. M. Floría and J. J. Mazo, Adv. Phys. 45, 505 (1996).

[16] F. Falo, L. M. Floría, P. J. Martínez, and J. J. Mazo, Phys. Rev. B 48, 7434 (1993).

[17] L. M. Floría and F. Falo, Phys. Rev. Lett. 68, 2713 (1992).

[18] B. Hu and J. Tekic, Phys. Rev. E 72, 056602 (2005).

[19] M. Inui and S. Doniach, Phys. Rev. B 35, 6244 (1987).

[20] M. J. Renné and D. Polder, Rev. Phys. Appl. 9, 25 (1974).

[21] J. R. Waldram and P. H. Wu, J. Low Temp. Phys. 47, 363 (1982).

[22] M. Remoissenet and M. Peyrard, Phys. Rev. B 29, 3153 (1984); M. Peyrard and M. Remoissenet, Phys. Rev. B 26, 2886 (1982).

[23] S. E. Hebboul and J. C. Garland, Phys. Rev. B 47, 5190 (1993).

[24] M. S. Rzchowski, L. L. Sohn, and M. Tinkham, Phys. Rev. B 43, 8682 (1991).

[25] S. Kim, B. J. Kim, and M. Y. Choi, Phys. Rev. B 52, 13536 (1995).

[26] S. J. Lee and T. C. Halsey, Phys. Rev. B 47, 5133 (1993).

[27] B. Hu and J. Tekić, Phys. Rev. E 75, 056608 (2007). 